ex Instituto Archaeologico Universitatis de Rolando Eötvös nominatae

C

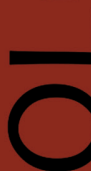

ப

$\varangle$

工

$\cup$

ه

$<$

in

ㄴ
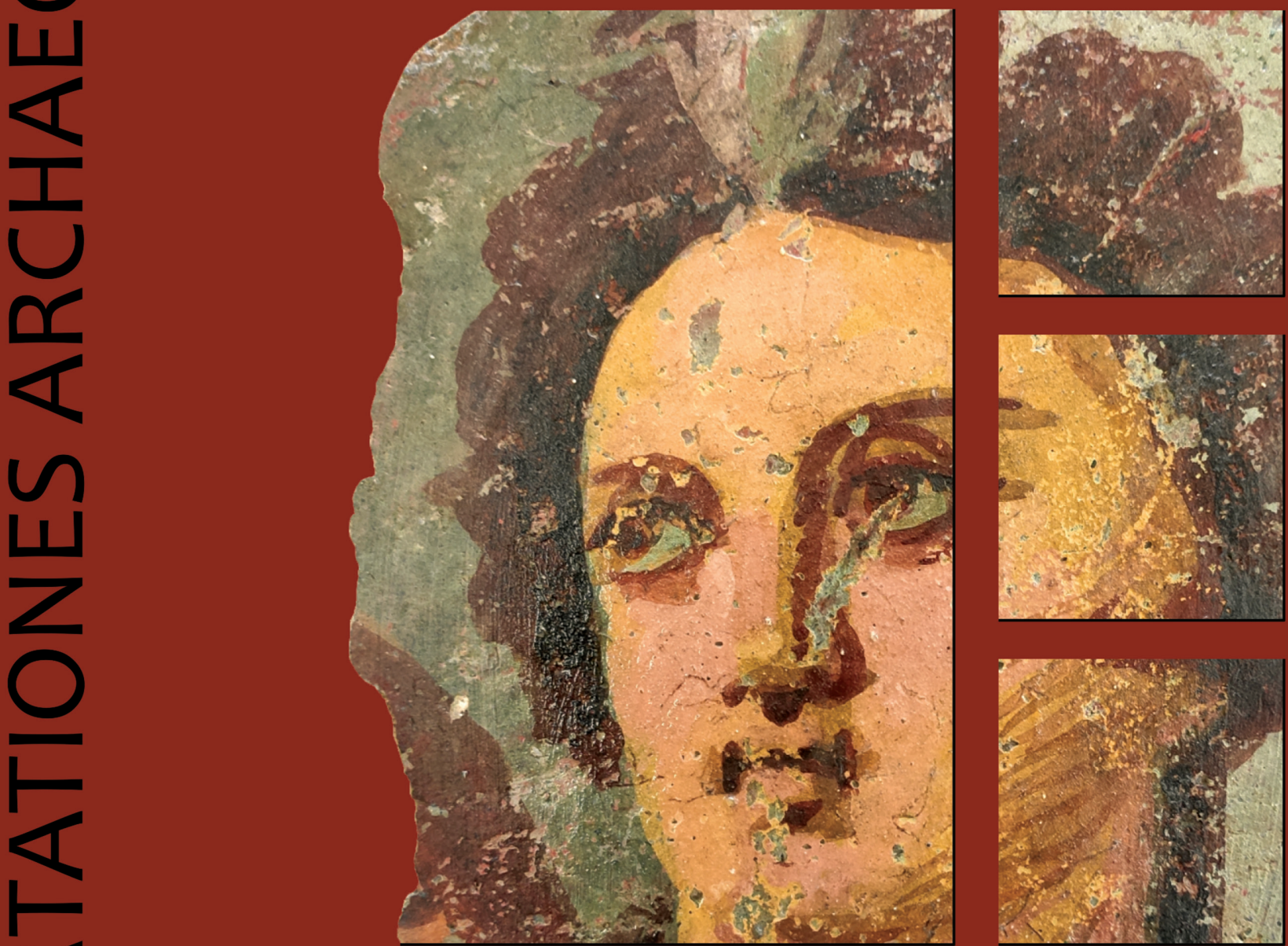

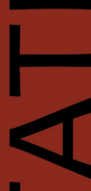

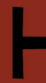

ח

Ш

un

n

0
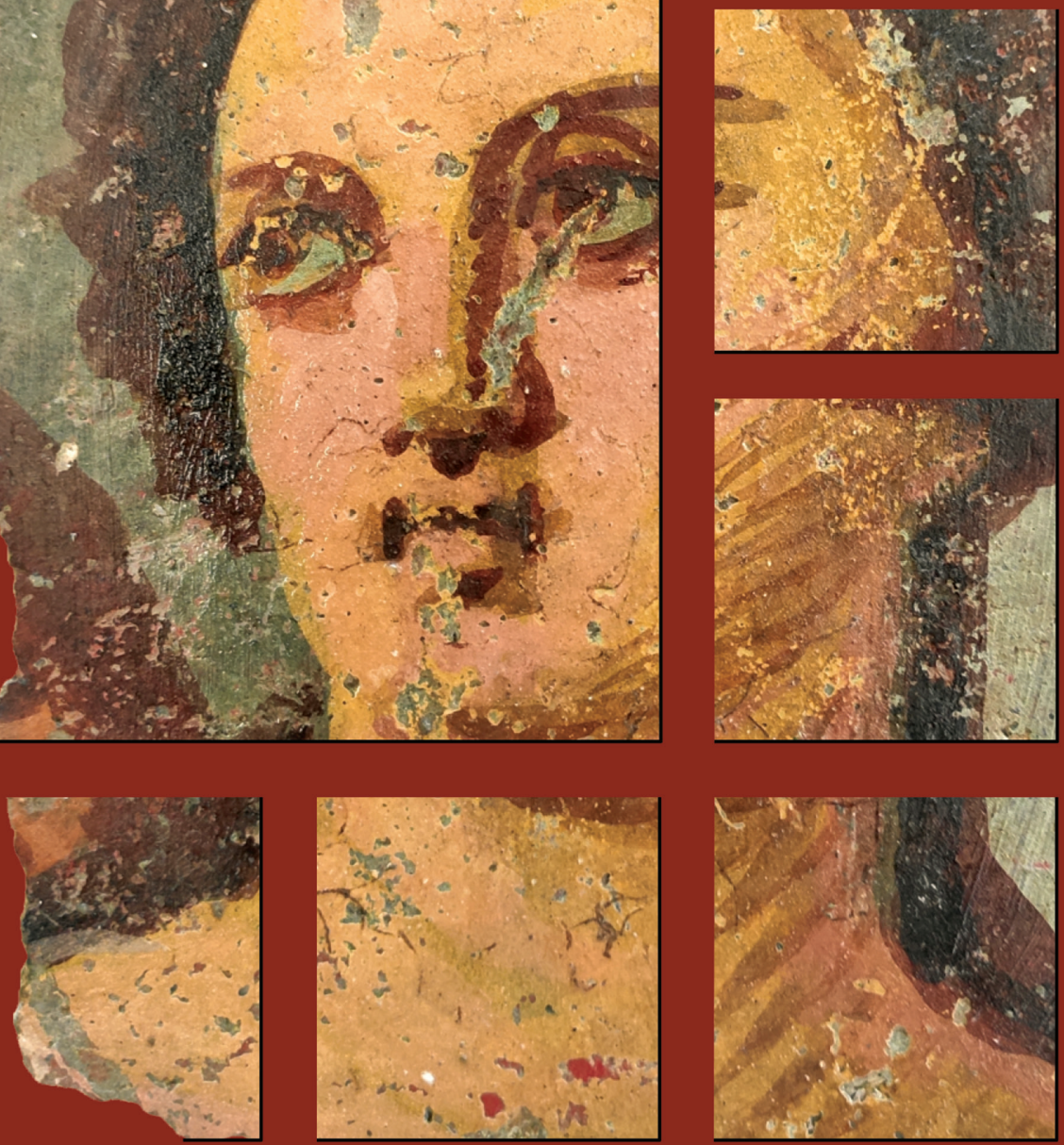

Serp 30 No. 8. 2020 


\section{Dissertationes Archaeologicae ex Instituto Archaeologico}

Universitatis de Rolando Eötvös nominatae Ser. 3. No. 8.

Budapest 2020 
Dissertationes Archaeologicae ex Instituto Archaeologico Universitatis de Rolando Eötvös nominatae Ser. 3. No. 8.

Editor-in-chief:

DÁvid Bartus

Editorial board:

LÁsZló BARTOSIEWICZ

LÁsZLÓ BORHY

ZOLTÁN CZAJLIK

IsTVÁN FELD

GÁBOR KALLA

PÁL RACZKY

MiKLÓS SZABÓ

Tivadar Vida

Technical editor:

Gábor VÁczi

Proofreading:

Szilvia BARTUS-SzÖLLŐsI

Zsófia KondÉ

Márton SZILÁGYI

Aviable online at http://ojs.elte.hu/dissarch

Contact: dissarch@btk.elte.hu

ISSN 2064-4574

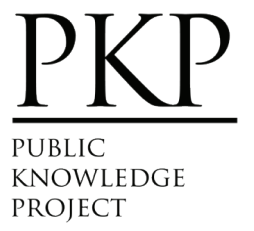

๑ ELTE Eötvös Loránd University, Institute of Archaeological Sciences

Layout and cover design: Gábor Váczi

Budapest 2020 


\section{CONTENTS}

\section{ARTICLES}

Maciej WAWRZCZAK - Zuzana KASENČÁKovÁ

Stará L'ubovña - Lesopark. Late Palaeolithic site and the problems associated with raw material mining

Attila PÉNTEK - Norbert FARAgó

Chipped stone assemblages from Schleswig-Holstein (North Germany) in the collection of the Institute of Archaeological Sciences - ELTE Eötvös Loránd University

Bence Soós 49

Middle Iron Age Cemetery from Alsónyék, Hungary

Tamás Szeniczey - Tamás Hajdu 107

Appendix - Results of the analysis of the Early Iron Age human remains unearthed at Alsónyék, Hungary

Lajos JuHÁsz - József Géza Kiss

Bound in bronze - a Roman bronze statuette of a barbarian prisoner

Csilla SÁRó

The fibula production of Brigetio: clay moulds

\section{Field Reports}

András Füzesi - Knut Rassmann - Eszter BÁnffy - Hajo Hoehler-Brockmann -

Gábor Kalla - Nóra Szabó - Márton SzIlágyi - Pál Raczky

Test excavation of the "pseudo-ditch" system of the Late Neolithic settlement complex at Öcsöd-Kováshalom on the Great Hungarian Plain

Gábor VÁczi - László RupNIK - Zoltán CZAJLIK - Gábor MEsterházy Bettina BitTner - Kristóf FÜlöP - Denisa M. LÖNHARdT - Nóra Szabó

The results of a non-destructive site exploration and a rescue excavation at the site of Pusztaszabolcs-Dohányos völgy északi part

Dávid BArtus - László Borhy - Szilvia JohÁczi - Emese SzÁmadó 181

Excavations in the legionary fortress of Brigetio in 2019 
Dávid BArtus - László Borhy - Emese SzÁmadó - Lajos Juhász - Bence Simon -

Ferenc Barna - Anita Benes - Szilvia Joháczi - Rita Olasz - Melinda Szabó

Excavations in Brigetio in 2020

\section{Thesis Abstracts}

Anett OszTÁs

The settlement history of Alsónyék-Bátaszék.

Complex analysis of its buildings in the context of the Lengyel culture

Csilla SzÁRAz

The region of the Zala and Mura Rivers (Zala County) in the Late Bronze Age.

Late Tumulus and Urnfield period

Ágnes KIRÁly

Human remains unearthed in settlement context from the Late Bronze Age -

Early Iron Age (Reinecke BD-HaB3) Northeastern Hungary

Gergely BóKA

Transformation of settlement history in the Körös Region in the period between the Late Bronze Age and the end of Iron Age

Gabriella G. DeLbó

Pottery production of the settlement complex of Brigetio

Adrienn Katalin BLAY

Die Beziehungen zwischen dem Karpatenbecken und dem Mediterraneum

von der II. Hälfte des 6. bis zum 8. Jahrhundert n. Chr. anhand Schmuckstücken

und Kleidungszubehör

Levente SAMU

293

Die mediterranen Kontakte des Karpatenbeckens in der Früh- und Mittel-

awarenzeit im Licht der Männerkleidung. Gürtelschnallen und Gürtelgarnituren

\section{REviEWS}

Gábor MESTERHÁZY

Czajlik, Z. - Črešnar, M. - Doneus, M. - Fera, M. - Hellmith Kramberger, A. Mele, M. (eds): Researching Archaelogical Landscapes Across Borders - Strategies,

Methods and Decisions for the 21th Century. Graz-Budapest, 2019. 


\title{
Die mediterranen Kontakte des Karpatenbeckens in der Früh- und Mittelawarenzeit im Licht der Männerkleidung
}

Gürtelschnallen und Gürtelgarnituren

\author{
Levente SAMU \\ Institute of Archaeological Sciences \\ ELTE Eötvös Loránd University \\ samulevente@gmail.com
}

\begin{abstract}
Abstract of PhD thesis submitted in 2020 to the Archaeology Doctoral Programme, Doctoral School of History, Eötvös Loránd University under the supervision of Tivadar Vida, Budapest and to the Institute of the Prehistory and Medieval Archaeology at the Albert-Ludwigs-University of Freiburg (Breisgau) under the supervision of Sebastian Brather.
\end{abstract}

\section{Thesen}

Das Ziel der Arbeit war, den mediterranen/byzantinischen Einfluss auf das Karpatenbecken in der zweiten Hälfte 6. und im 7. Jh. n. Chr. anhand den Gürtelgarnituren und Gürtelschnallen des 7. Jhs. zu entdecken und zu analysieren. Es wurde auch gezielt, die Intensität der Kontakte zu prüfen und möglichst neue Aspekte zur Erforschung dieser Frage hineinzubringen und ein mehr komplexes Bild über dieses archäologische Phänomen zu geben. Die byzantinische, bzw. mediterrane Welt hatte einen allgemeinen Einfluss auf seine Umgebung. Da die Region des Karpatenbeckens ${ }^{1}$ im 6.-7. Jh. ein „Randgebiet “ ${ }^{{ }_{2}}$ des Byzantinischen Reiches war, ist es wesentlich die Intensität ihrer Kontakte in Richtung des Mediterraneums zu erforschen. Diese Arbeit wurde auf den Arbeiten von Jörg Drauschke, ${ }^{3}$ Éva Garam, ${ }^{4}$ Mechthild Schulze-Dörrlamm, ${ }^{5}$ Tivadar Vida ${ }^{6}$ und vergleichbaren Studien über die Vermittlung der unterschiedlichen Produkte/ Rohstoffe mediterraner Provenienz basiert.

Neben den physikalischen Eigenschaften (die Größe, das Material, die Farbe, die Verknüpfung, die Beschläge, die Steineinlagen, die Gehänge, die Inschriften, die Gürtelstruktur, die Verzierung) wurden die möglichen Bedeutungen des Gürtels behandelt. Es wurde zusammengefasst, dass ein Gürtel eine symbolische Funktion (bei den Gürtelschenkungen, in den zwischenmenschlicher Beziehungen, im Ordensleben, im Eheritual, als göttliches Attribut, bei den Initiationsriten, als Rangabzeichen, in der Kirche, im Militär und andere Ämte) tragen konnte. ${ }^{7}$ Als Schmuckobjekt war es auch wesentlich. Eine magische Funktion konnte auch assoziiert

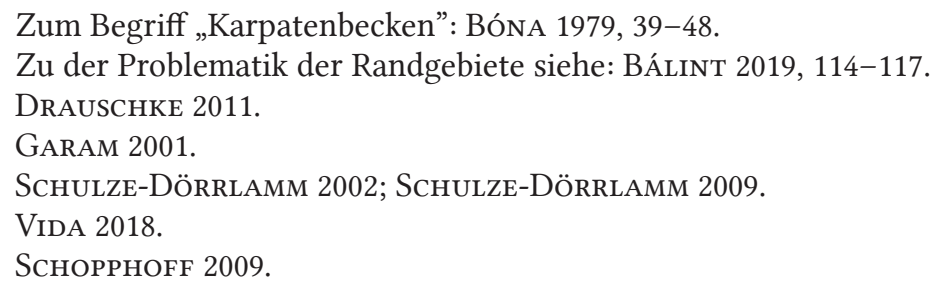


werden - ein Gürtel kann Kraft geben, er kann schützen (Apotropäum) und als wundertätige Reliquie angesehen werden. Die byzantinischen Quellen beweisen, dass der Gürtel nicht einfach ein Prestigegegenstand war, aber es wurde zu bestimmten Positionen geknüpft. Im Fall der Gesellschaft des awarenzeitlichen Karpatenbeckens kann es aber nicht beweist werden, wir können nur anhand der Zahl der Gürtelgarnituren, bzw. die fernöstlichen und byzantinischen Parallelen vermuten, dass es ein sehr wichtiger Prestigegenstand war, was auch in der Wiederaufteilung der Güter und damit in der Aufrechterhaltung der Abhängigkeiten eine wichtige Rolle spielen konnte.

Mit der Berücksichtigung der Probleme der Interpretation wurde es relativ früh entschieden, dass nicht nur die schon „ausgewählten“ und bekannten „Gegenstände byzantinischer Herkunft" analysiert werden, sondern das ganze Gürtelmaterial des Karpatenbeckens aufgenommen wird und damit die möglichen neuen, bisher nicht bewussten Kontakte aufgedeckt werden können. Der Autor hatte sich entschieden alle Gegenstände, die eine mediterrane/ byzantinische Ornamentik oder von diesen beeinflussten Darstellungen tragen als die Hinterlassenschaft der mediterranen/byzantinischen Kontakte zu betrachten. Eine klare Trennung zwischen den „Originalien“ und den „Nachahmungen“ ist kaum möglich, deutlich wichtiger ist der Anspruch diese Gegenstände zu kopieren - auch in solchen Fällen, wenn die originelle Bedeutung einer Darstellung schon sicherlich verloren wurde. Es ist sehr wahrscheinlich, dass dieselbe Darstellung andere Bedeutung für den byzantinischen Träger als dem Träger im awarenzeitlichen Karpatenbecken hatte. Anderes Erträgnis war, dass mit dieser Methode die klaren Proportionen mediterranen und anders interpretierten Gegenständen gezeigt werden konnten und überhaupt die genaue Zahl der Gürtelzierden analysiert werden konnte. Aus der Sicht des Autors sind diese Daten wesentlich um die mediterrane und byzantinische (und übrigens alle andere) Einflüsse auf das Material zu analysieren und zu interpretieren.

Es wurde - anhand der ungestörten Gräber - auch gezeigt, dass die sogenannten vielteiligen Gürtelgarnituren in sehr großer Variabilität auftreten. Die Zahl der Gürtelzierden in diesen Garnituren bewegt sich meistens zwischen 1-20. Selbst die Variabilität der Gürtelzierden innerhalb diesen Garnituren ist sehr groß. Daher ist es ganz problematisch, idealtypischen Garnituren zu suchen. Sie sind eher die Ausnahmen, als der Regel. Diese Erscheinung wurde vor allem von der Grabsitte - die in den verschiedenen Gemeinden abweichend war - beeinflusst. Wir müssen damit rechnen, dass das, was wir im Mehrheit der Gräber sehen, ist kein vollständiges Bild. Es ist - anhand den Daten - sehr wahrscheinlich, dass nicht immer, bzw. meistens keine vollständigen Gürtel in den Gräber gelangt sind. Idealtypen kommen seltener vor und die Zahl der „Idealtypen“ kann deutlich höher sein, als wir es bei heutigem Forschungsstand vermuten.

Eine grundsätzliche Systematisierung der Gürtelzierden des Karpatenbeckens wurde durchgeführt. Die häufigste Form der Riemenzungen sind die parallelen Seiten mit bogiger Endung. Relativ häufig tritt aber die gespitzte Endung auch auf. Die Gestaltung der Seiten, wie auch der Endung ist aber sehr variabel. Die häufigsten Beschlagformen sind die verschiedenen rundlichen und rechteckigen Formen, bzw. die Wappenform. Bei den übrigen Beschlagformen ist es auch charakteristisch, dass die Form von dem abgetragenen Motiv determiniert ist.

Die Verbreitung der Gürtelgarnituren verändert sich zwischen der ersten und zweiten Hälfte des 7. Jhs. Im allgemeinen kann es festgestellt werden, dass in der ersten Hälfte des 7. Jhs. einige Regionen starker gesondert werden können, in der zweiten Hälfte des 7. Jhs. wird das 
Gesamtbild mehr zersplittert. Die Zentren, die für die erste Hälfte des 7. Jhs. typisch waren, teils bleiben, teils ändern. Manche Gebiete, die aus der Sicht der Verbreitung dichter sind, waren schon auch in der Römerzeit intensiv benutzt - sie waren für diese Raumnutzung geographisch determiniert. Bedenkliche Veränderungen kann man ab der zweiten Hälfte des 7. Jhs. beobachten: die früheren Verbreitungen verändern sich - die Verbreitung der Fundorte wird viel mehr diffus, in den früher leeren Gebiete werden auch Fundorte auftreten. Im Hintergrund dieser Wandlungen stehen wahrscheinlich der Wandel der Gesellschaft, die Demographie, die neue Raumnutzung, und ein Wandel des Wirtschaftssystems.

Es wurde auch beobachtet, dass zwischen der ersten und zweiten Hälfte des 7. Jhs. die Zahl der Gürtelzierden in den nördlichen Gebieten deutlich größer wird als es früher war und es relativ höher wird, als in den südlichen Gebieten. Es kann mehrfach interpretiert werden, eine mögliche Lösung ist, dass die neu gegründeten Gräberfelder das Gesamtbild beeinflussen. Die Zusammensetzung der Gürtel kann auch nicht außer Acht gelassen werden. Es ist auf jeden Fall sicher, dass ab der zweiten Hälfte des 7. Jhs. mehrere neue Gräberfelder in den nördlichen Regionen des Karpatenbeckens geöffnet wurden. In diesen Gräberfeldern ist der Anteil der mit Gürtel Bestatteten auch relativ hoch. Diese Veränderung kann auf den Interpolationskarten beobachtet werden. Die ergriffenen Prozesse haben ein neues Beweis dafür gebracht haben, dass der Kaganat ein dynamisches System war, im ständigen Wechsel und dass die Besiedlung des Karpatenbeckens zwischen letzten Drittel des 6. Jhs. und Ende 7. Jhs. deutlich verändert hat.

Die mediterranen Schnallen wurden auch aufgenommen und analysiert. Neue Typen wurden gesondert und alle nummeriert von 1 bis 49. Deutlich größer ist die Variabilität der Gürtelschnallen ab der zweiten Hälfte des 6. Jhs. bis Mitte des 7. Jhs. Es wurde aber auch bemerkt, dass es leider nicht direkt auf die Intensität der Kontakte übersetzt werden kann. Wenn wir die Zahl der späteren Gürtelschnallen - auch von anderen Gebieten - genauer anschauen, wird es sofort bemerkbar, dass ihre Zahl ist deutlich niedriger. Es handelt sich hier also über keine lokale Erscheinung, sondern um eine allgemeine, überregionale Erscheinung. Die Tendenz, was die Tabellen zeigen, ist sehr bemerkenswert, da beiden Schnallensammlungen (aus dem RGZM und aus dem Karpatenbecken) eine größere Variabilität der Schnallentypen ab Ende 6. bis Mitte 7. Jhs. zeigen. Diese Erscheinung scheint eine allgemeine Tendenz des Mittelmeerraums und damit des Byzantinischen Reiches zu sein, was sich in der Hinterlassenschaft des Karpatenbeckens zwischen Mitte 6. und Ende 7. Jhs. widerspiegelt. Es kann daher so bewertet werden, dass unser Arbeitsgebiet in dem bestimmten Zeitraum im mediterranen Austauschssystem - gewissermaßen - integriert war, es gab ein Bedarf für diese Gegenstände. Die Zahl der Gürtelschnallen und ihre Variabilität kann aber nicht direkt als der Abdruck der Kontaktintensität interpretiert werden. Ab Ende 6. bis Mitte 7. Jhs. zeigen die Schnallen die größte Vielfältigkeit.

Allgemeine Tendenzen bezüglich der Ornamentik konnten auch festgelegt werden. Ein deutlicher Unterschied zwischen der ersten und zweiten Hälfte des 7. Jhs. ist, dass in der zweiten Hälfte des Jahrhunderts zahlreiche Motive in stark geometrischen Form ausgeführt werden. Mehrere neue Motive treten auch auf, aber auch schon früher bekannten Motiven werden auch geometrisch (oft ganz gewinkelt) ausgeführt. Neben diesen geometrisch ausgeführten Formen bleiben aber auch die „bogigen“ Formen in der Mode, sie sind v. a. die aus der Spätawarenzeit gut bekannten pflanzlichen Motive und einige Flechtbände. Eine Geometrisierung 
der Motive ist aber nicht nur für die zweite Hälfte des 7. Jhs. typisch. Wie es gezeigt wurde, es war schon ab Mitte des 6. Jhs. geübt - die Maskendarstellungen sind auch ein Ergebnis dieser Tendenzen. Diese Erscheinungen - d. h. die Schematisierung und Geometrisierung - sind grundsätzlich in allen Zeitphasen anwesend, was sie resultierten ist aber deutlich unterschiedlich, es hängt eng von dem herrschenden Motivschatz ab. Daher sind die Motive der zweiten Hälfte des 6. Jhs. und der zweiten Hälfte des 7. Jhs. deutlich unterschiedlich.

Die maskenartigen und damit zusammenhängenden Motive wurden kategorisiert und kartiert. Sie zeigen starke Kontakte in die Richtung der unteren Donau und der Krim, aber sie treten in weiten Gebieten zwischen Italien und dem Kaukasus auf. Es ist auch augenfällig, dass die verschiedenen Maskenmotive und ihre Varianten verschiedene Verbreitungen zeigen. Was besonders bemerkenswert ist, dass einige Typen völlig aus dem unteren Donauraum fehlen (z. B. die dreieckförmige Durchbrüche und die schlüssellochförmige Durchbrüche), dagegen einige Typen konzentrieren sich direkt auf dieses Gebiet (z. B. die geometrische und schematische Durchbrüche und der U-förmige Durchbruch). Es hängt wahrscheinlich mit der Vermittlung und Distribution zusammen und meiner Meinung nach spricht es dafür, dass eine Kommunikation zwischen dem Pontus-Gebiet und dem Karpatenbecken nicht nur durch dem unteren Donauraum entstanden hat, sondern durch die kürzere Wege der Karpaten.

Bei der Analyse der Motive des ausgehenden 6. - ersten Hälfte 7. Jhs. wurde als Ausgangspunkt die Punkt-Komma Ornamentik genommen, da sie einerseits als zentrale Verzierung auftreten, andererseits wurden sie als Umrahmung genutzt und so knüpfen sie zahlreiche Motive zusammen - die wir allgemein als mediterrane Motive interpretieren. Es wurde versucht, zeitgleiche Parallelen aus dem Mediterraneum zu finden. Die Motive, die wir aus den Gürtelzierden kennen, treten an verschiedenen Gegenstandtypen in ganz verschiedenen Formen auf. Das Ziel war hier einfach zu zeigen, dass dieser Motivschatz in einem breiten Raum allgemein bekannt und genutzt war. Beispiele wurden aus dem Feld der Steinschnitzerei, der Textilkunst, der Beinschnitzerei und der Münzprägung gezogen. Der Motivschatz wurde relativ detailliert analysiert, die förmlichen Variationen und die verschiedenen Ausführungen wurden auch gezeigt. Es wurde hingewiesen, dass bestimmte Prozesse im Material beobachtet werden können, so wie die Schematisierung, Geometrisierung, bzw. Zerfall bestimmten Motive - aber alle diese entstandene Varianten und Stilen können auf dasselbe Ornamentik zurückgeführt werden. Es wurde auch gezeigt, wie können bestimmte - oft figural aussehende - Motive auf die pflanzliche Ornamentik zurückgeführt werden.

Die Kontexte der mit Gürtelgarnituren versehenen Gräber wurde auch betrachtet, dabei die Männer-, Frauen- und Kindergräber auch. Am Anfang wurde es gezeigt, dass die Beigaben der Frauen- und Männergräber relativ stark abweichen. Im Allgemeinen konnte es festgestellt werden, dass die Palette der Beigaben der Frauengräber mit Gürtelzierden ist fortgesetzt einfacher geworden: weniger Beigabentypen traten in größeren Zahl auf. Interessant ist dieser Hinsicht, dass diese Erscheinung bei den Männern nicht beobachtet wurde. Die Variabilität der Beigaben blieb ständig, nur die Zusammensetzung geänderte sich.

Aussagen über die Intensität der Kontakte können daher so formuliert werden, dass zwar das Karpatenbecken im ständigen Kontakt mit der mediterranen Welt war und die Tendenzen dieser Welt ihre Abdrücke in dem Karpatenbecken zwischen Mitte 6. und Ende 7. Jhs. gehabt haben, bestimmte Erscheinungen nur für dieses Gebiet typisch sind. Eine ist die eigenartige $\mathrm{Zu}-$ sammensetzung des Materials - nicht alle Schnallen- und Gürteltypen gelangten hier, die aber 
doch gekommen sind, zeigen eine große Vielfältigkeit, die nur für das Arbeitsgebiet typisch ist. Die Intensität der Kontakte - nach der Meinung des Authors - wurde vielmehr durch die Vielfalt der Objekt- und Motivtypen, als durch die einfache Zahl der Gegenstände widergespiegelt. Die Vielfalt dauert - wahrscheinlich wegen einer zwanghaften Repräsentation - ganz bis zum mittleren Drittel des 7. Jhs. aus. Die Veränderungen im Gürtelmaterial laufen selbstverständlich nicht allein, deutliche Dynamiken können in der räumlichen Verteilung der Güter beobachtet werden und im Hintergrund laufen auch die Veränderungen der Siedlungsstruktur. Wenn wir für die Aussage der Verbreitungskarten glauben können, werden die das Karpatenbecken erreichenden Vermittlungslinien ab der zweiten Hälfte des 7. Jhs auch begrenzter oder mindestens weniger benutzt. Selbstverständlich können die im Gürtelmaterial beobachteten Erscheinungen parallel mit aus den schriftlichen Quellen bekannten Ereignissen ausgewertet werden, das Gürtelmaterial kann aber nur solche Ereignisse widerspiegeln, die langfristigen Auswirkungen auf der Gesellschaft haben. Solche Widerspiegelungen können die Vielfältigkeit der Gürteltypen und Verzierungen, die „Überrepräsentation“ auch im Gürtelmaterial in den reichen Gräber, und der Wandel der Gürtelgarnituren ab der zweiten Hälfte des 7. Jhs. sein.

\section{Literatur}

BÁLInT, Cs. 2019: The Avars, Byzantium and Italy. A Study in Chronology and cultural History. Varia Archaeologica Hungarica 31, Budapest.

BóNA, I. 1979: Régészetünk és Kelet-Európa. MTA II. Osztályának Közleményei 28, 39-48.

Drauschke, J. 2011: Zwischen Handel und Geschenk - Studien zur Distribution von Objekten aus dem Orient, aus Byzanz und aus Mitteleuropa im östlichen Merowingerreich. Freiburger Beiträge zur Archäologie und Geschichte des ersten Jahrtausends 14. Rahden/Westf.

Garam, É. 2001: Funde byzantinische Herkunft in der Awarenzeit vom Ende des 6. bis zum Ende des 7. Jahrhunderts. Monumenta Avarorum Archaeologica 5. Budapest.

Schopphoff, C. 2009: Der Gürtel. Funktion und Symbolik eines Kleidungsstücks in Antike und Mittelalter. Wien. doi: 10.7788/9783412333638

Schulze-DörRlamm, M. 2002: Byzantinische Gürtelschnallen und Gürtelbeschläge im Römisch-Germanischen Zentralmuseum. Teil 1: Die Schnallen ohne Beschläg, mit Laschenbeschläg und mit festem Beschläg des 5. bis 7. Fahrhunderts. Römisch Germanisches Zentralmuseum - Kataloge Vor- und Frühgeschichtlicher Altertümer 30/1, Mainz.

Schulze-Dörrlamm, M. 2009: Byzantinische Gürtelschnallen und Gürtelbeschläge im Römisch-Germanischen Zentralmuseum. Teil 2: Die Schnallen mit Scharnierbeschläg und die Schnallen mit angegossenem Riemendurchzug des 7. bis 10. Jahrhunderts. Römisch Germanisches Zentralmuseum Kataloge Vor- und Frühgeschichtlicher Altertümer 30/2, Mainz.

VIDA, T. 2018: A sztyeppei, a Bizánci és a Meroving birodalmak között. Kulturális változások a Kárpátmedence nyugati felén a 6-7. században. Academic thesis (manuscript). Budapest. 
